\title{
Impact of the introduction of the model for end-stage liver disease system on the low volume liver transplant centers: a multicenter study
}

Tae Yun Lee, Young Chul Yoon

Department of Surgery-Hepatobiliary, The Catholic University of Korea, Incheon St. Mary's Hospital, Incheon, Korea

Background: The shortage of donor organs in transplantation is the biggest obstacle to organ transplantation. From June 1 , 2016, the Korean organ transplant standard was converted to the model for end-stage liver disease (MELD) system. This study aims to clarify the differences in deceased donor liver transplantation (DDLT) before and after the introduction of the MELD system in three low volume centers in Gyeongin area.

Methods: From June 2013 to May 2019, the study was conducted retrospectively with adult patients undergoing deceased donor liver transplantation at Incheon St. Mary Hospital, Bucheon Soonchunhyang Hospital, and Incheon Gil Hospital.

Results: Of the 431 registered patients, a total of 87 patients who underwent DDLT were studied. Before June 2016 (before the MELD system) was designated as the Child-Turcotte-Pugh (CTP) group, and later as the MELD group. Finally, the CTP group was 39 patients, and the MELD group was 48 patients. There were no statistical differences in sex and age of recipients and donors. The primary disease was alcoholic liver disease in the MELD group. There was no statistical difference between transplant zone distribution and blood type. In outcome, the MELD group showed remarkably short transplant waiting time. There was no statistically difference in survival rates between the two groups.

Conclusions: After the introduction of the MELD system, alcoholic liver disease was the most common primary disease, and the waiting period for transplantation was shortened. There was no statistically significant difference in hospitalization period and survival rate between the two groups. On this study, the introduction of the MELD system can be considered the advantage of being more distributed to critical, severe patients.

Corresponding author: Young Chul Yoon

E-mail:k07yyc@catholic.ac.kr

(c) The Korean Society for Transplantation

This is an Open Access article distributed under the terms of the Creative Commons Attribution Non-Commercial License (http://creativecommons.org/licenses/by-nc/4.0/) which permits unrestricted non-commercial use, distribution, and reproduction in any medium, provided the original work is properly cited. 